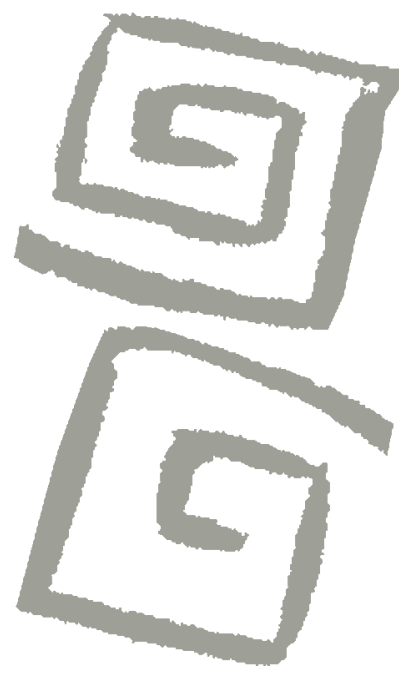

\title{
Excesivo rastreo de osteoporosis en mujeres menores de 65 años: estudio de corte transversal
}

\author{
Excessive osteoporosis screening in women under 65 \\ years: a cross-sectional study
} María de las Nieves Ganiele', Sergio Adrián Terrasa² ${ }^{1}$ Karin Silvana
Kopitowski

${ }^{1}$ Médica de familia. Médica asociada. Docente, Departamento de Medicina, Instituto Universitario del Hospital Italiano de Buenos Aires, Argentina. $\square$ (iD)

${ }^{2}$ Médico de familia. Investigador Formado. Médico de Planta, Servicio de Medicina Familiar y Comunitaria, Hospital Italiano de Buenos Aires. Docente, Departamento de Salud Pública, Instituto Universitario del Hospital Italiano de Buenos Aires. Argentina. $\triangle$ (iD)

${ }^{3}$ Médica de familia. Jefa, Servicio de Medicina Familiar y Comunitaria, Hospital Italiano de Buenos Aires. Directora, Departamento de Investigación, Instituto Universitario del Hospital Italiano de Buenos Aires, Argentina. $\square$ (iD)
RESUMEN El rastreo de osteoporosis en mujeres con bajo riesgo de fractura (sobreuso) puede conducir a sobrediagnóstico, tratamiento inapropiado y medicalización. El objetivo de este trabajo fue determinar la proporción de mujeres de 45 a 64 años afiliadas a un plan de medicina prepaga de Buenos Aires, Argentina, que realizaron al menos una densitometría ósea de cadera durante 2011 y no cumplían criterios para el rastreo. Se realizó un estudio observacional de corte transversal. Se identificaron 4.310 mujeres de este rango etario que se realizaron una densitometría ósea, entre las que se seleccionó una muestra aleatorizada de 401 mujeres y se obtuvieron datos completos para $178 \mathrm{mu}$ jeres. Para determinar si el rastreo era apropiado se utilizaron dos criterios: 1) tener un riesgo de fractura a 10 años mayor que una mujer de 65 años (regla FRAX ${ }^{\circledR}$ ); 2) presentar al menos un factor de riesgo de fractura. Un 86,5\% de las densitometrías óseas fueron realizadas en mujeres cuyo riesgo estimado por $\mathrm{FRAX}^{\circledR}$ no superaba el umbral mínimo recomendado, constatándose osteoporosis en el 5,8\% y osteopenia en el 61,0\%. En relación con el segundo criterio, el 49,4\% no presentaba siquiera un factor de riesgo, documentándose osteoporosis en el 3,4\%, y osteopenia en el 62,5\%. Los resultados muestran que al menos la mitad de las mujeres no cumplía con los criterios de rastreo.

PALABRAS CLAVES Osteoporosis; Tamizaje Masivo; Mal Uso de Servicios de Salud; Argentina.

\begin{abstract}
Overuse of osteoporosis screening in women at low risk of fracture may lead to overdiagnosis, inappropriate treatment and medicalization. The objective of this work was to estimate the proportion of women aged 45 to 64 enrolled in a private health insurance plan in Buenos Aires undergoing hip dual-energy x-ray absorptiometry (DXA) in 2011 without meeting osteoporosis screening criteria. In this cross-sectional study, 4310 women of this age range that had undergone a hip DXA were identified. A randomly selected subgroup of 401 women was then assessed for the presence of risk factors for osteoporosis and complete data were retrieved for 178 women. Appropriate screening was defined by two criteria: 1) having a 10-year fracture risk higher than that of a 65 -year old woman (estimated using the FRAX ${ }^{\circledR}$ tool); 2) having at least one risk factor for fracture. It was found that $86.5 \%$ of the women who underwent hip DXA did not exceed the minimum 10-year fracture risk threshold required for screening; $5.8 \%$ of them had osteoporosis and $61.0 \%$ osteopenia. According to the second criterion, $49.4 \%$ had no risk factors, $3.4 \%$ of these women had osteoporosis and $62.5 \%$ osteopenia. The results show that at least half the women screened did not meet osteoporosis screening criteria.
\end{abstract}

KEY WORDS Osteoporosis, Mass Screening, Health Services Misuse, Argentina. 


\section{INTRODUCCIÓN}

A medida que crece la expectativa de vida, aumenta la proporción de la población proclive a fracturarse ${ }^{(1)}$, fundamentalmente, la columna vertebral, la muñeca y la cadera. La fractura de cadera se asocia a pérdida de la independencia y disminución de la calidad y expectativa de vida, ya que el $20 \%$ de quienes las sufren requieren atención domiciliaria, solo cerca del $40 \%$ recuperan totalmente su nivel de independencia previo y, en cuanto a la probabilidad de morir al año(2), superan en un $15 \%$ a la población general de su misma edad. En promedio, los costos directos del tratamiento de cada paciente que sufre una fractura de cadera se estiman, en Argentina, en 3.800 dólares estadounidenses, y representan anualmente, a nivel nacional, un total de 130 millones dólares ${ }^{(3)}$.

La Organización Mundial de la Salud (OMS) definió como fractura osteoporótica por fragilidad aquella causada por un trauma leve (fuerza equivalente a una caída de la propia altura o menos), que sería insuficiente para fracturar un hueso normal. Por otro lado, en 1994, un panel de expertos propuso criterios diagnósticos de osteoporosis densitométrica basados en la determinación de la densidad mineral ósea por densitometría ósea, lo que permite pensar a la osteoporosis densitométrica como un factor de riesgo de la fractura osteoporótica por fragilidad, caracterizado por una baja masa ósea y un deterioro de su microarquitectura ${ }^{(4)}$. En forma operativa, se dice que una persona tiene el factor de riesgo de osteoporosis densitométrica cuando su masa ósea se encuentra 2,5 desviaciones estándar por debajo del promedio observado en la población sana de 30 años (edad en la que se alcanza la masa ósea máxima). Se reporta como un puntaje T menor o igual a $-2,5^{(5)}$.

Las estrategias de rastreo pretenden identificar a las mujeres asintomáticas con alta probabilidad de fractura osteoporótica por fragilidad para asesorarlas sobre la prevención de caídas y, eventualmente, si su perfil de riesgo lo amerita, el tratamiento antiresortivo. Diferentes sociedades científicas, que basan sus consensos en revisiones sistemáticas, concuerdan en que todas las mujeres deben realizar una densitometría ósea de rastreo a los 65 años, y comenzar a edades más tempranas solo si su probabilidad basal de fractura está aumentada ${ }^{(6,7,8,9)}$.

Existen dos criterios principales para detectar a la subpoblación de mujeres menores de 65 años con riesgo aumentado de fractura osteoporótica por fragilidad:

1. Utilizar la regla de predicción clínica Fracture Risk Assessment Tool (FRAX $\left.{ }^{\circledR}\right)$, que combina información de diferentes variables asociadas con la probabilidad de desarrollar estas fracturas. A partir de los resultados de una revisión sistemática y metaanálisis de datos primarios de nueve cohortes prospectivas, los creadores incorporaron las siguientes variables en la calculadora FRAX ${ }^{\circledR}$ : sexo, edad, índice de masa corporal, antecedentes personales de fractura, fractura de cadera en padres, uso crónico de corticoides, tabaquismo, alcoholismo y otras causas de osteoporosis secundaria. La calculadora FRAX ${ }^{\circledR}$ considera las características epidemiológicas locales de diferentes países, le otorga a cada factor un coeficiente independiente que refleja su capacidad predictiva y funciona con o sin datos de la densitometría ósea del cuello femoral. Se utiliza para determinar quiénes exceden el umbral de riesgo, y puede ser aplicada en personas de ambos sexos de 40 a 90 años. Esta regla considera como umbral para el rastreo el riesgo de fractura a 10 años de una mujer blanca de 65 años sin factores de riesgo (recomendación tipo B) ${ }^{(7,10)}$ que, en Argentina, es un $6,9 \%{ }^{(11,12)}$.

2. La presencia de al menos un factor de riesgo de los mencionados previamente ${ }^{(13,14)}$.

Sin embargo, existe consenso en que las fracturas osteoporóticas por fragilidad tienen una etiología múltiple. En su camino causal es destacable el rol de las caídas vinculadas al desacondicionamiento muscular por falta de ejercicio y/o malnutrición; déficits del equilibrio y de los reflejos posturales por 
consumo de fármacos, envejecimiento y pérdida de movilidad articular; problemas visuales; falta de adaptación de las viviendas a las necesidades de quienes van envejeciendo; aislamiento social; etc. Dada la naturaleza multifactorial de las fracturas y sus consecuencias (resultado final que pretendemos evitar), al igual que otros autores ${ }^{(15,16)}$, consideramos que la medición de la densidad mineral ósea y la posterior prescripción de fármacos -sin considerar la probabilidad global de la fractura osteoporótica por fragilidad del paciente en cuestión y sin abordar en forma multidisciplinaria los eslabones causales de las caídas, probablemente por influencia de las estrategias de promoción de la industria farmacéutica- vienen ocupando un rol exageradamente central en la toma de decisiones que procuran prevenir la ocurrencia de fracturas, ya que este factor de riesgo solo explica una pequeña parte de la multicausalidad de estos eventos ${ }^{(15,17)}$. Esto ha conducido, por un lado, a que durante las dos últimas décadas el diagnóstico de osteoporosis densitométrica y las decisiones de tratamiento de los pacientes se hayan basado principalmente en los resultados de la densitometría ósea y, por otro lado, a que algunos grupos poblacionales estén siendo "sobre-rastreados" con densitometría ósea, con la consiguiente posibilidad de sobrediagnóstico de osteoporosis densitométrica, medicalización, etiquetamiento e ineficiente asignación de recursos ${ }^{(18,19,20)}$.

Sobre la base de la coincidencia de esta descripción con nuestra apreciación clínica, se decidió realizar este trabajo con el objetivo principal de determinar la proporción de mujeres de 45 a 64 años afiliadas a un plan de medicina prepaga (Hospital Italiano de Buenos Aires) que realizaron al menos una densitometría ósea de cadera y raquis durante 2011 y no cumplían criterios de rastreo de osteoporosis densitométrica (rastreo inapropiado). A su vez, se plantearon como objetivos secundarios: describir la distribución de los resultados de dichas densitometrías óseas, determinar la proporción de prescripción de tratamiento antiresortivo en el subgrupo de mujeres que no cumplían criterios de rastreo de osteoporosis y analizar como potencial predictor del pedido adecuado de densitometría ósea que el cuidado de salud estuviera coordinado por el médico de cabecera vs. médico especialista (endocrinología, reumatología y/o ginecología).

\section{MATERIAL Y MÉTODOS}

Se realizó un estudio de corte transversal llevado a cabo en el Hospital Italiano de Buenos Aires (hospital universitario privado, situado en la Ciudad Autónoma de Buenos Aires y en el Gran Buenos Aires), que posee un seguro privado de salud y que atiende, principalmente, a población de clase media. Se solicitó al Servicio de Informática Médica un listado de mujeres afiliadas al seguro médico prepago que, al 31 de diciembre de 2011, tuvieran entre 45 y 64 años de edad $(n=19.799)$ y hubieran realizado al menos una densitometría ósea durante ese año $(n=4.310)$. De ese listado de 4.310 mujeres se realizó una aleatorización simple, en la cual los números identificatorios del listado inicial fueron ordenados aleatoriamente a través del programa True Random Number Service. De este ranking aleatorizado se seleccionaron las primeras 401 mujeres, de las cuales efectivamente se logró obtener información completa (a través de la historia clínica y de entrevista telefónica) de 178 mujeres.

Para la categorización de las densitometrías óseas se utilizaron los criterios diagnósticos de la OMS, que establece osteoporosis densitométrica ante un puntaje $\mathrm{T}$ menor $\mathrm{o}$ igual a $-2,5$, osteopenia con un puntaje entre $-2,5$ y -1, y normalidad con un puntaje igual o mayor a -1. Se consideró que el rastreo fue inapropiado ante la falta de cumplimiento de alguno de los siguientes criterios:

1.Presencia de un riesgo de fractura a 10 años menor al 6,9\% (riesgo basal de una mujer argentina de 65 años sin factores de riesgo de fractura osteoporótica por fragilidad) según la regla de predicción clínica FRAX ${ }^{\circledR}$. 
2.Presencia de al menos un factor de riesgo de fractura osteoporótica por fragilidad ${ }^{(7,10)}$.

El resultado de las densitometrías óseas, la prescripción de tratamiento farmacológico (bifosfonatos, calcio y vitamina D) y las variables demográficas (edad, sexo, peso, talla e índice de masa corporal) fueron obtenidas de la historia clínica electrónica. La presencia de factores de riesgo de fractura osteoporótica por fragilidad, como antecedente propio de una fractura osteoporótica en la adultez de columna, cadera y/o muñeca; antecedente de fractura de cadera en familiar de primer grado; tabaquismo activo; consumo crónico de glucocorticoides (uso diario vía oral por al menos tres meses consecutivos); artritis reumatoidea; consumo excesivo de alcohol -tres o más dosis ("tragos") al día (8 a 10 g de alcohol)-; causas de osteoporosis secundaria como diabetes tipo I (insulino-dependiente), osteogénesis imperfecta del adulto, hipertiroidismo, hipogonadismo o menopausia prematura (antes de los 45 años), malnutrición o malabsorción crónica o hepatopatía crónica, fue obtenida a través de una entrevista telefónica, previa lectura del consentimiento informado y aceptación oral por parte de la entrevistada.

Se definió que la paciente tenía cuidados médicos coordinados por un médico de cabecera si al menos el $50 \%$ del total de las consultas habían sido con su médico de cabecera durante los 48 meses previos a la realización de la densitometría ósea en cuestión (índice de prestador habitual de cuidado mayor a 0,5$)^{(21)}$. Además se registró el número de consultas a especialistas en ginecología, reumatología y endocrinología en ese mismo período.

El análisis estadístico se llevó a cabo utilizando el software Stata 8.0. Dados los antecedentes reportados en la bibliografía especializada y asumiendo una tasa de rastreo inapropiado cercana al 50\%, hemos estimado que se necesitaba una muestra final de, al menos, 164 observaciones (pacientes) para obtener una estimación de dicha tasa con un intervalo de confianza de hasta 7,5\% de semi-amplitud ${ }^{(19)}$. Se realizó un análisis multivariable a través de un modelo de regresión logística para evaluar la asociación entre la realización apropiada de una densitometría ósea y el haber tenido un cuidado coordinado por el médico de cabecera.

\section{Aspectos éticos de la investigación}

En todo momento se trabajó de manera confidencial; la base de datos de los pacientes se encontraba protegida por contraseña, y solo tenían acceso a ella los miembros del equipo de investigación. El estudio se realizó de acuerdo a las normas regulatorias de la investigación humana a nivel nacional y de la provincia de Buenos Aires, en concordancia con la Resolución 1480/2011 del Ministerio de Salud de la Nación y las recomendaciones en investigación médica en seres humanos de la Declaración de Helsinki de 1964 con todas sus enmiendas y las guías de Buenas Prácticas Clínicas de la Organización Panamericana de la Salud ${ }^{(22)}$.

Asimismo, el protocolo fue aprobado por el Comité de Ética de Protocolos de Investigación del Hospital Italiano de Buenos Aires (código 1884).

\section{RESULTADOS}

De la muestra aleatorizada inicialmente de 401 mujeres, se logró contactar telefónicamente a 190. De las contactadas, 187 dieron su consentimiento para la entrevista y, de ellas, 178 habían realizado una densitometría ósea de cadera y de raquis. La media de edad de las mujeres contactadas fue de 58,5 y su índice de masa corporal de $25,5 \mathrm{~kg} / \mathrm{m}^{2}$.

De los factores de riesgo relevados a partir de las entrevistas telefónicas los más prevalentes fueron: tabaquismo en un $25,8 \%$ y menopausia precoz en un $19,2 \%$, mientras que el $13,2 \%$ refirió que su padre o madre había tenido una fractura de cadera. No se evidenciaron diferencias clínicas ni estadísticamente significativas entre la subpoblación que contestó la encuesta telefónica y la que 
Tabla 1. Comparación de las variables estudiadas entre la población contactada con datos completos y la no contactada y/o con datos incompletos. Buenos Aires, Argentina, 2011.

\begin{tabular}{|c|c|c|c|c|c|}
\hline \multirow[t]{2}{*}{ Variables } & \multicolumn{2}{|c|}{$\begin{array}{c}\text { Contactadas } \\
\text { con datos completos } \\
(\mathrm{n}=178)\end{array}$} & \multicolumn{2}{|c|}{$\begin{array}{c}\text { No contactadas y/o } \\
\text { con datos incompletos } \\
(\mathrm{n}=223)\end{array}$} & \multirow[t]{2}{*}{ Valor de $p$} \\
\hline & $\bar{x}$ & DS & $\bar{x}$ & DS & \\
\hline Edad en años & 58,5 & 4,5 & 58,3 & 4,8 & 0,712 \\
\hline Índice de masa corporal & 25,5 & 4,2 & 25,5 & 4,4 & 0,959 \\
\hline Consultas con médico de cabecera & 5,6 & 6,1 & 4,9 & 5,7 & 0,224 \\
\hline \multirow[t]{2}{*}{ Consultas con especialistas } & 3,0 & 3,3 & 3,4 & 3,9 & \multirow[t]{2}{*}{0,285} \\
\hline & $\mathrm{M}_{\mathrm{e}}$ & RI & $\mathrm{M}_{\mathrm{e}}$ & RI & \\
\hline \multirow[t]{2}{*}{ Consultas totales } & 7,5 & 3-11 & 7 & $3-11$ & \multirow[t]{2}{*}{0,657} \\
\hline & $\mathrm{n}$ & $\%$ & $\mathrm{n}$ & $\%$ & \\
\hline Tabaquismo & 45 & 25,6 & 50 & 22,4 & 0,464 \\
\hline \multicolumn{6}{|l|}{ Densitometría } \\
\hline \multicolumn{6}{|l|}{ Raquis } \\
\hline Normal & 69 & 38,8 & 86 & 39,5 & \multirow{3}{*}{0,898} \\
\hline Osteopenia & 85 & 47,8 & 106 & 48,6 & \\
\hline Osteoporosis & 24 & 13,5 & 26 & 11,9 & \\
\hline \multicolumn{6}{|l|}{ Cadera } \\
\hline Normal & 56 & 31,5 & 77 & 36,5 & \multirow{3}{*}{0,373} \\
\hline Osteopenia & 111 & 62,4 & 117 & 55,5 & \\
\hline Osteoporosis & 11 & 6,2 & 17 & 8,1 & \\
\hline $\begin{array}{l}\text { Cuidados de salud coordinados } \\
\text { por médico de cabecera }\end{array}$ & 118 & 66,3 & 145 & 65,0 & 0,790 \\
\hline
\end{tabular}

Fuente: Elaboración propia.

$\overline{\mathrm{x}}=$ Media $; \mathrm{DS}=$ Desvío estándar $; \mathrm{M}_{\mathrm{e}}=$ Mediana $; \mathrm{RI}=$ Rango intercuartílico.

no lo hizo, respecto de su edad, índice de masa corporal, prevalencia de tabaquismo, resultados de la densitometría ósea, consultas médicas totales, con el médico de cabecera y especialistas (Tabla 1).

Como respuesta al objetivo principal, la proporción del total de las densitometrías óseas que fueron realizadas en mujeres con bajo riesgo de fractura osteoporótica por fragilidad a 10 años, y basándonos en el primer criterio de rastreo propuesto, el $86,5 \%$ [IC 95\% $(80,6 ; 91,2)]$ de las densitometrías óseas fueron realizadas en mujeres que no cumplían el criterio de rastreo de la regla $\mathrm{FRAX}^{\circledR}$ (no superaban el riesgo umbral de fractura osteoporótica por fragilidad que en nuestro país es del 6,9\% a 10 años, por lo que son consideradas de bajo riesgo).

En cuanto a la proporción del total de las densitometrías óseas que fueron realizadas en mujeres que no tenían siquiera un solo factor de riesgo de fractura osteoporótica por fragilidad, tomando en cuenta el segundo criterio propuesto, fue del 49,4\% [IC 95\% $(41,9 ; 57,0)]$ (Tabla 2).

De las 154 mujeres que no cumplían los criterios de rastreo con densitometría ósea de

Tabla 2. Proporción de mujeres que realizaron densitometría ósea según criterios de rastreo. Buenos Aires, Argentina, 2011.

\begin{tabular}{lccc} 
Criterio de rastreo & $\mathbf{n}$ & $\%$ & IC 95\% \\
$\begin{array}{lccc}\text { No cumplían criterio FRAX } \\
\text { No presentaban factores de riesgo }\end{array}$ & 154 & 86,5 & 80,$6 ; 91,2$ \\
\hline
\end{tabular}

Fuente: Elaboración propia.

FRAX $^{\circledR}=$ Fracture Risk Assessment Tool; IC 95\% = Intervalo de confianza del 95\%. 
Tabla 3. Distribución de los resultados de las densitometrías óseas según criterios de rastreo. Buenos Aires, Argentina, 2011.

\begin{tabular}{lrrc|} 
Criterios de rastreo & $\mathbf{n}$ & $\%$ & IC $\mathbf{9 5 \%}$ \\
$\begin{array}{l}\text { No cumplían criterio FRAX } \\
\text { (n=154) }\end{array}$ & & & \\
$\quad$ Normal & 51 & 33,1 & 25,$7 ; 41,1$ \\
$\quad$ Osteopenia & 94 & 61,0 & 52,$9 ; 68,8$ \\
$\quad$ Osteoporosis & 9 & 5,8 & 2,$7 ; 10,1$ \\
$\begin{array}{l}\text { No presentaban factores } \\
\text { de riesgo (n=88) }\end{array}$ & & & \\
$\quad$ Normal & 30 & 34,1 & 24,$3 ; 45,0$ \\
$\quad$ Osteopenia & 55 & 62,5 & 51,$5 ; 72,6$ \\
$\quad$ Osteoporosis & 3 & 3,4 & 0,$71 ; 9,6$ \\
\end{tabular}

Fuente: Elaboración propia.

FRAX $^{\circledR}=$ Fracture Risk Assessment Tool; IC 95\% = Intervalo de confianza del $95 \%$.

acuerdo con la regla FRAX ${ }^{\circledR}$, se diagnosticó osteoporosis densitométrica al 5,8\% y osteopenia densitométrica al $61,0 \%$, y de las 88 mujeres que no presentaban factores de riesgo al 3,4\% y al $62,5 \%$ respectivamente (Tabla 3 ).

En el $35,1 \%$ de las 154 mujeres que no cumplían con criterios FRAX ${ }^{\circledR}$ y en el $35,2 \%$ de las 88 que no cumplían los criterios de rastreo con densitometría ósea se constató la prescripción de bifosfonatos en la historia clínica electrónica (Tabla 4).
Tabla 4. Proporción de mujeres con bajo riesgo de fractura osteoporótica por fragilidad según criterios de rastreo, en las que se documentó la prescripción de tratamiento farmacológico. Buenos Aires, Argentina, 2011.

$\begin{array}{lccc}\begin{array}{l}\text { Prescripción de tratamiento } \\ \text { farmacológico }\end{array} & \mathbf{n} & \% & \text { IC 95\% } \\ \begin{array}{l}\text { No cumplían criterio FRAX } \\ \text { (n=154) }\end{array} & & & \\ \quad \begin{array}{l}\text { Bifosfonatos (con o sin calcio y/o } \\ \text { vitamina D) }\end{array} & 54 & 35,1 & 27,3 ; 43,2 \\ \quad \text { Calcio y/o vitamina D solamente } & 52 & 33,8 & 26,4 ; 41,8 \\ \begin{array}{l}\text { No presentaban factores de } \\ \text { riesgo (n=88) }\end{array} & & & \\ \quad \begin{array}{l}\text { Bifosfonatos (con o sin calcio y/o } \\ \text { vitamina D) }\end{array} & 31 & 35,2 & 25,3 ; 46,1 \\ \quad \text { Calcio y/o vitamina D solamente } & 25 & 28,4 & 19,3 ; 39,0 \\ & & & \end{array}$

Fuente: Elaboración propia.

FRAX $^{\circledR}=$ Fracture Risk Assessment Tool; IC 95\% = Intervalo de confianza del $95 \%$

Como era de esperarse, las mujeres con bajo riesgo de fractura por $\mathrm{FRAX}^{\circledR}$ tendieron a ser significativamente más jóvenes que las que superaban dicho umbral. A través del análisis de regresión logística no se observó una asociación estadísticamente significativa entre tener cuidados de salud coordinados por el médico de cabecera y la chance de

Tabla 5. Distribución de la edad, el índice de masa corporal y los cuidados de salud de forma coordinada de acuerdo al cumplimiento de criterios de rastreo con densitometría ósea de cadera. Buenos Aires, Argentina, 2011.

\begin{tabular}{|c|c|c|c|c|c|c|c|}
\hline Criterios de rastreo & $\%$ & $\bar{x}$ & IC $95 \%$ & $\%$ & $\bar{x}$ & IC $95 \%$ & Valor de $p$ \\
\hline \multicolumn{8}{|l|}{$\begin{array}{l}\text { Riesgo por regla de } \text { FRAX }^{\circledR} \\
\text { Sí }(n=24) ; \text { no }(n=154)\end{array}$} \\
\hline Edad en años & - & 61,8 & 60,$4 ; 63,3$ & - & 58,1 & 57,$3 ; 58,8$ & 0,0001 \\
\hline IMC & - & 24,7 & 23,$1 ; 26,1$ & - & 25,6 & 24,$9 ; 26,3$ & 0,307 \\
\hline Cuidados de salud coordinados & 70,8 & - & - & 65,6 & - & - & 0,613 \\
\hline \multicolumn{8}{|l|}{$\begin{array}{l}\text { Presencia de factores de riesgo } \\
\text { Sí }(n=90) ; \text { no }(n=88)\end{array}$} \\
\hline Edad en años & - & 58,5 & 57,$5 ; 59,5$ & - & 58,7 & 57,$8 ; 59,6$ & 0,353 \\
\hline IMC & - & 25,2 & 24,$3 ; 26,0$ & - & 25,8 & 24,$8 ; 26,7$ & 0,366 \\
\hline Cuidados de salud coordinados & 65,6 & - & - & 67,1 & - & - & 0,833 \\
\hline
\end{tabular}

Fuente: Elaboración propia.

FRAX $^{\circledR}=$ Fracture Risk Assessment Tool. $\bar{x}=$ Media; IMC $=$ Índice de masa corporal. 
que la densitometría correspondiera a una mujer que cumplía criterios de rastreo por FRAX $^{\circledR}[O R$ 1,34; IC $95 \%(0,53 ; 3,43)]$ o con al menos un factor de riesgo de fractura osteoporótica por fragilidad [OR 0,95; IC 95\% $(0,51 ; 1,76)]$ (Tabla 5).

\section{DISCUSIÓN}

Los resultados de nuestra investigación nos permitieron estimar con buena precisión tanto la proporción de densitometrías óseas realizadas durante 2011 en mujeres que no cumplían mínimos criterios de rastreo, como la distribución de sus resultados y la proporción de quienes tenían prescripto algún fármaco implicado en el metabolismo fosfocálcico en la historia clínica electrónica.

Nuestros resultados coinciden con los observados en países que no pertenecen a Latinoamérica, como en la investigación publicada por Schnatz et al. sobre una población de 600 mujeres posmenopáusicas atendidas entre 2007 y 2009 en una institución privada de radiología situada en Hartford, Connecticut, EE.UU. Estos autores documentaron que el $41,3 \%$ de las densitometrías óseas habían sido realizadas en personas que no cumplían los criterios de rastreo propuestos por la North American Menopause Society ${ }^{(19)}$. La pertinencia en la solicitud de densitometría ósea también fue evaluada en los pacientes de la cohorte ESOSVAL en Valencia (hombres y mujeres de 50 a 74 años), en la cual uno de cada diez pacientes a quienes se les había solicitado una densitometría ósea tampoco cumplía con los criterios de rastreo de diversas asociaciones médicas ${ }^{(23)}$.

En concordancia con estos resultados, un estudio de casos y controles realizado en Buenos Aires informó que el seguimiento por médicos especialistas aumentaría la chance de la solicitud inapropiada de densitometría ósea para rastreo ${ }^{(18)}$. Sin embargo, nuestra investigación es la primera en Argentina en la que, más allá de los datos secundarios consignados en la historia clínica, se recurrió a fuentes primarias de información, como fueron los Ilamados telefónicos a las mujeres que habían realizado la densitometría ósea con el fin de evitar rotular como "sobre-rastreada" a una mujer con un riesgo mayor real al documentado en su historia clínica.

Merece un comentario especial el haIlazgo de osteopenia densitométrica en el $61 \%$ y de osteoporosis densitométrica en el $5,8 \%$ de la población que tenía bajo riesgo de fractura osteoporótica por fragilidad y que, por lo tanto, no cumplía criterios de rastreo apropiados según los consensos vigentes y no deberían haber realizado la densitometría ósea ${ }^{(11,12,13,14)}$. Vale destacar que el evento a prevenir es la fractura osteoporótica por fragilidad y que las decisiones terapéuticas deberían tomarse en función del riesgo de este tipo de lesiones y no solo a consecuencia de valores densitométricos aislados ${ }^{(15,16,24)}$. Estas afirmaciones están respaldadas por los hallazgos publicados por Kanis et al., quienes documentaron que la probabilidad de fractura depende mucho más de la edad que del valor aislado de densidad mineral ósea ${ }^{(25)}$. Dicho de otra manera, aunque una mujer con bajo riesgo basal de fractura osteoporótica por fragilidad tenga una densitometría ósea con valores bajos de densidad mineral de acuerdo a la distribución que se usa como referencia (población sana de 30 años), es muy poco probable que se beneficie al recibir tratamiento antiresortivo, ya que el impacto del tratamiento dependerá de su probabilidad postest de presentar una fractura osteoporótica por fragilidad, probabilidad que surge luego de agregar la información de la densitometría ósea a la de los factores de riesgo que ya estaban presentes antes de realizarla. Por lo tanto, es falaz concluir que una persona con bajo riesgo basal de fractura osteoporótica por fragilidad tiene osteoporosis densitométrica porque tiene un estudio cuyos resultados arrojan un valor alejado de la media de referencia (que además es cuestionable, ya que es por lo menos sospechoso usar como referencia a una población veinte años más joven que la población a la que se aplica masivamente dicho test: la que supera los 50 años de edad) y es muy probable que, 
del $60 \%$ de densitometrías con hallazgos "anormales", la mayoría sean episodios de sobrediagnóstico, que aumentarán la probabilidad de sobretratamiento y etiquetamiento. Como sostienen Zárate et al., no existe respaldo para el tratamiento farmacológico de las personas con osteoporosis densitométrica y ausencia de factores de riesgo de fractura osteoporótica por fragilidad, y menos aún para el tratamiento de pacientes con osteopenia aislada ${ }^{(26)}$, mientras que Sosa Henríquez et al. sostienen que el concepto de osteopenia se trata de una construcción teórica que fue desarrollada con fines epidemiológicos y de utilidad clínica cuestionable ${ }^{(27)}$. Por eso consideramos que haber hallado osteopenia en alrededor de un $60 \%$ de las mujeres con bajo riesgo de fractura osteoporótica por fragilidad solo puede haber conducido a generarles preocupación, gastos y/o riesgo de efectos adversos de fármacos, sin que exista evidencia de que ese hallazgo pueda redundar en beneficios potenciales para su salud ${ }^{(24,28)}$.

\section{Limitaciones de nuestra investigación}

Con relación al análisis de posibles predictores no hemos detectado asociación entre la presencia de "sobre-rastreo" (o rastreo inapropiado en mujeres con bajo riesgo de fractura osteoporótica por fragilidad) y los cuidados de salud coordinados por el médico de cabecera. Sin embargo, nuestra investigación no contaba con el poder suficiente para detectarla, ya que su tamaño muestral había sido calculado en función de nuestro objetivo principal. Esta investigación fue realizada en una población afiliada al subsistema privado de salud del área metropolitana de la Ciudad Autónoma de Buenos Aires y del Gran Buenos Aires, lo que nos obliga a ser cautos antes de extrapolar sus conclusiones a otros grupos poblacionales. Nuestra tasa de respuesta fue ligeramente inferior $(44,4 \%)$ a la reportada en estudios de base poblacional de otros países $(56 \%)$, sin embargo, no pareciera haber diferencias sistemáticas entre el subgrupo contactado y el que no lo fue ${ }^{(29)}$ (Tabla 1).

\section{CONCLUSIÓN}

Alrededor de la mitad de las mujeres a quienes se les realizó una densitometría ósea no reunían criterios para el rastreo de osteoporosis, aun aplicando los más intervencionistas. Consideramos que la prescripción inadecuada de la densitometría ósea puede conducir a que mujeres de bajo riesgo global de fractura osteoporótica por fragilidad sean etiquetadas como "enfermas" de osteoporosis/osteopenia, y sometidas a tratamientos innecesarios y potencialmente riesgosos, más allá de la consiguiente dilapidación de recursos humanos y económicos en salud a los que se les podría dar una mejor utilización.

\section{AGRADECIMIENTOS}

Agradecemos a Georgina Bonavita, alumna de la carrera de medicina del Instituto Universitario del Hospital Italiano de Buenos Aires por la colaboración en la realización de entrevistas telefónicas. Agradecemos al Servicio de Informática Médica del Hospital Italiano por brindarnos la base de datos inicial. Agradecemos al Dr. Eduardo Durante por la revisión de una versión preliminar del artículo.

\section{REFERENCIAS BIBLIOGRÁFICAS}

1. Schurman L, Bagur A, Claus-Hermberg $H$, Messina OD, Negri AL, Sánchez A, et al. Guías 2012 para el diagnóstico, la prevención y el tratamiento de la osteoporosis. Medicina (Buenos Aires). 2013;73(1):55-74.

2. World Health Organization. What evidence is there for the prevention and screening of osteoporosis? [Internet]. Copenhagen: WHO; 2006 [citado 18 may 2015]. Disponible en: http://goo.gl/IIxYlj. 
3. Spivacow FR, Sánchez A. Epidemiology, costs, and burden of osteoporosis in Argentina. Archives of Osteoporosis. 2010;5(1):1-6.

4. Consensus Development Conference. Diagnosis, prophylaxis and treatment of osteoporosis. American Journal of Medicine. 1993;94(6):646-650.

5. Kanis JA, Melton LJ, Christiansen C, Johnston CC, Khaltaev N. The diagnosis of osteoporosis. Journal of Bone and Minereral Research. 1994;9(8):1137-1141.

6. National Osteoporosis Foundation. Clinician's guide to prevention and treatment of osteoporosis. Washington DC: National Osteoporosis Foundation; 2014.

7. U.S. Preventive Services Task Force. Screening for osteoporosis: U.S. preventive services task force recommendation statement. Annals of Internal Medicine. 2011;154(5):356-364.

8. Qaseem A, Snow V, Shekelle P, Hopkins R Jr, Forciea MA, Owens DK, Clinical Efficacy Assessment Subcommittee of the American College of Physicians. Pharmacologic treatment of low bone density or osteoporosis to prevent fractures: a clinical practice guideline from the American College of Physicians. Annals of Internal Medicine. 2008;149(6):404-415.

9. Lim LS, Hoeksema LJ, Sherin K, ACPM Prevention Practice Committee. Screening for osteoporosis in the adult U.S. population: ACPM position statement on preventive practice. American Journal of Preventive Medicine. 2009;36(4):366-375.

10. Kanis JA, Oden A, Johansson H, McCloskey E. FRAX ${ }^{\circledR}$ y la evaluación de la probabilidad de fractura: introducción. Revista Metabolismo Óseo y Mineral. 2010;8(1):15-18.

11. Claus-Hermberg $H$, Bagur A, Messina OD, Negri $A L$, Schurman L, Sánchez A. FRAX ${ }^{\circledR}$ : un nuevo instrumento para calcular el riesgo absoluto de fracturas a 10 años. Medicina (Buenos Aires). 2009;69(5):571-575.

12. FRAX ${ }^{\circledR}$ WHO Fracture Risk Assessment Tool [Internet]. World Health Organization Collaborating Centre for Metabolic Bone Diseases [citado 15 may 2015]. Disponible en: http://goo.gl/CPiWYR.

13. The North American Menopause Society. Management of osteoporosis in postmenopausal women: 2006 position statement of the NAMS. Menopause. 2006;13:340-367.

14. Papaioannou A, Morin S, Cheung AM, Atkinson S, Brown JP, Feldman S, et al. 2010 clinical practice guidelines for the diagnosis and management of OP in Canada. CMAJ. 2010;182(17):1864-1873.

15. Moniyan R, Cassels A. Selling sickness: How the world's biggest pharmaceutical companies are turning us all into patients. New York: Nation Books; 2006.

16. Järvinen TLN, Michaëlsson K, Jokihaara J, Collins GS, Perry TL, Mintzes B, et al. Overdiagnosis of bone fragility in the quest to prevent hip fracture. BMJ. 2015;350:h2088.

17. Järvinen TLN, Sievänen $H$, Khan KM, Heinonen A, Kannus P. Shifting the focus in fracture prevention from osteoporosis to falls. BMJ. 2008;336:124-126.

18. Ortuño $M$, Clavijo $M$, García Allende $N$, Popoff F, Aizpurua F, Izcovich A, Catalano H. Uso y sobreuso de la densitometría ósea como método de screening de osteoporosis. Revista Argentina de Medicina. 2013;1(1):13-16.

19. Schnatz PF, Marakovits KA, Dubois $M$, O'Sullivan DM. Osteoporosis screening and treatment guidelines: are they being followed? Menopause. 2011; 18:1072-1078.

20. Gérvas J, Pérez Fernández M. Cribados: una propuesta de racionalización. Gaceta Sanitaria. 2013;27:372-373.

21. Reid R, Haggerty J, McKendry R. Defusing the confusion: Concepts and measures of continuity of care [Internet]. Ottawa: Fondation Canadienne de la Recherche sur les Services de Santé; 2002 [citado 10 may 2015]. Disponible en: http://goo. gl/4e5NPB.

22. Organización Panamericana de la Salud. Buenas Prácticas Clínicas: Documento de las Américas. [Internet]. OPS/OMS; 2005 [citado 10 may 2015]. Disponible en: https://goo.gl/T1 rxWl.

23. Hurtado I, Sanfélix-Gimeno G, Baixauli-Pérez C, Peiró S, Sanfélix-Genovés J. Impact on the population of different bone mineral density testing criteria and appropriateness of densitometries in the ESOSVAL cohort, Spain. Journal of Clinical Endocrinology and Metabolism. 2014;99(1):142-150.

24. U.S. Department of Health and Human Services. Bone Health and Osteoporosis: A Report of the Surgeon General. Rockville, MD: U.S. Department of Health and Human Services, Office of the Surgeon General; 2004.

25. Kanis J, Johnell O, Oden A, Dawson A, De Laet C, Jonsson B. Ten year probabilities of osteoporotic fracture according to BMD and diag- 
nostic thresholds. Osteoporosis International. 2001;12(12):989-995.

26. Zárate A, Saucedo R. Controversia sobre el manejo de la osteopenia. Acta Médica Grupo Angeles. 2003;1(4):241-242.

27. Sosa Henríquez M, Gómez de Tejada Romero MJ. El término osteopenia y el riesgo de fractura. Anales de Medicina Interna (Madrid). 2006;23(4):151-152.
28. Khosla S, Melton L. Osteopenia. New England Journal of Medicine. 2007;356:2293-2300. doi: 10.1056/NEJMcp070341.

29. Galán I, Rodríguez Artalejo F, Zorrilla B. Reproducibilidad de un cuestionario telefónico sobre factores de riesgo asociados al comportamiento y las prácticas preventivas. Gaceta Sanitaria. 2004;18:118-128.

\section{FORMA DE CITAR}

Ganiele MN, Terrasa SA, Kopitowski KS. Excesivo rastreo de osteoporosis en mujeres menores de 65 años: estudio de corte transversal. Salud Colectiva. 2016;12(3):443-452. doi: 10.18294/sc.2016.841.

Recibido: 12 de enero de 2016 | Versión final: 29 de mayo de 2016 | Aprobado: 20 de junio de 2016

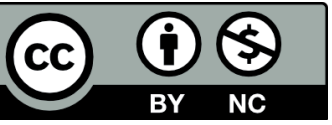

Este obra está bajo una licencia de Creative Commons Reconocimiento-NoComercial 4.0 Internacional. Reconocimiento - Permite copiar, distribuir y comunicar públicamente la obra. A cambio, se debe reconocer y citar al autor original. No Comercial - Esta obra no puede ser utilizada con finalidades comerciales, a menos que se obtenga el permiso.

http://dx.doi.org/10.18294/sc.2016.841 